\title{
Effects of oblique incidence and colliding pulses on laser-driven proton acceleration from relativistically transparent ultrathin targets
}

\author{
J. Ferri ${ }^{\oplus 1, \dagger, ~ E . ~ S i m i n o s ~}{ }^{2}$, L. Gremillet ${ }^{\oplus 3,4}$ and T. Fülöp ${ }^{\oplus 1}$ \\ ${ }^{1}$ Department of Physics, Chalmers University of Technology, SE-41296 Göteborg, Sweden \\ ${ }^{2}$ Department of Physics, University of Gothenburg, SE-41296 Göteborg, Sweden \\ ${ }^{3}$ CEA, DAM, DIF, F-91297 Arpajon, France \\ ${ }^{4}$ Université Paris-Saclay, CEA, LMCE, 91680 Bruyères-le-Châtel, France
}

(Received 1 April 2020; revised 13 July 2020; accepted 14 July 2020)

The use of ultrathin solid foils offers optimal conditions for accelerating protons to high energies from laser-matter interactions. When the target is thin enough that relativistic self-induced transparency sets in, all of the target electrons get heated to high energies by the laser, which maximizes the accelerating electric field and therefore the final ion energy. In this work, we first investigate how ion acceleration by ultraintense femtosecond laser pulses in transparent $\mathrm{CH}_{2}$ solid foils is modified when turning from normal to oblique $\left(45^{\circ}\right)$ incidence. Due to stronger electron heating, we find that higher proton energies can be obtained at oblique incidence but in thinner optimum targets. We then show that proton acceleration can be further improved by splitting the laser pulse into two half-pulses focused at opposite incidence angles. An increase by $\sim 30 \%$ in the maximum proton energy and by a factor of $\sim 4$ in the high-energy proton charge is reported compared to the reference case of a single normally incident pulse.

Key words: intense particle beams, plasma simulation

\section{Introduction}

Laser-driven proton acceleration using ultraintense pulses has been an active field of research in the past two decades (Tikhonchuk 2010; Daido, Nishiuchi \& Pirozhkov 2012; Macchi, Borghesi \& Passoni 2013). The strong electric fields produced in the interaction of the ultraintense laser pulse with solid targets can accelerate protons to high energies on acceleration distances of only a few microns. This has led to the implementation or exploration on small-scale facilities of a large variety of applications, such as radiography (Romagnani et al. 2005), generation of warm dense matter (Patel et al. 2003; Pelka et al. 2010), inertial confinement fusion (Roth et al. 2001), nuclear physics (McKenna et al. 2003) or proton therapy (Bulanov \& Khoroshkov 2002). The most investigated, and

†Email address for correspondence: julien.ferri@chalmers.se 
routinely exploited, mechanism is the so-called target normal sheath acceleration (TNSA), which benefits from a relatively easy implementation (Clark et al. 2000; Maksimchuk et al. 2000; Snavely et al. 2000; Wilks et al. 2001). TNSA, however, yields maximum ion energies $E_{\max }$ that scale rather weakly with the laser intensity $I_{0}\left(E_{\max } \propto I_{0}^{\alpha}\right.$, with $\alpha \simeq 0.5-1$ depending on the laser duration) (Zeil et al. 2010). This limitation motivates the investigation of alternative, possibly more efficient schemes, e.g. radiation pressure (or light-sail) acceleration (Esirkepov et al. 2004; Kar et al. 2012), shock acceleration (Silva et al. 2004; Haberberger et al. 2012) (with $E_{\max } \propto I_{0}$ ), directed Coulomb explosion (Bulanov et al. 2008) or breakout afterburner (Yin et al. 2006).

The possibility of employing several laser pulses to control the preplasma formation and improve the general efficiency of the TNSA process has been studied in experiments (Markey et al. 2010; Scott et al. 2012; Brenner et al. 2014; Ferri et al. 2018). In particular, in a recent work, we studied the influence of splitting the laser pulse in two pulses containing each half of the total energy in a TNSA set-up (Ferri, Siminos \& Fülöp 2019). In that work, the half-pulses were simultaneously focused onto a few- $\mu \mathrm{m}$ thick target, but at opposite incidence angles. Through particle-in-cell (PIC) simulations, we showed that the proton energy could be increased in the two-pulse scheme by approximately $80 \%$ with lasers impinging at $\pm 45^{\circ}$ angles onto a foil target with sub-wavelength density scale length. Such enhancement was found to result from a more efficient, Brunel-type vacuum heating of the electrons, due to the laser interference, and the ensuing suppression of the mitigating $v \times B$ force (with $v$ the particle velocity and $B$ the magnetic field), at the target surface (Geindre, Audebert \& Marjoribanks 2006).

While those results are very promising for TNSA experiments, notably those performed at ultrahigh laser contrast (Ceccotti et al. 2007), they also suggest possible improvements for other acceleration set-ups, especially those involving ultrathin (nanometric) target foils. Previous studies indeed demonstrated that, in the case of femtosecond laser pulses, proton energies are maximized for target thicknesses such that relativistic self-induced transparency (RSIT) occurs near the laser peak (Esirkepov, Yamagiwa \& Tajima 2006; d'Humières et al. 2013; Brantov et al. 2015). It is therefore worth exploring whether the use of two interfering half-pulses in this regime could significantly enhance the hot-electron generation, and hence lower the RSIT threshold.

In this work, therefore, we investigate, by means of PIC numerical simulations, how splitting the laser pulse into two half-pulses focused at opposite incidence angles affects the interaction with nanometric foils. For the purpose of establishing a reference case, we first investigate the dynamics of $\mathrm{CH}_{2}$ foils driven by a single ultraintense laser pulse under normal or oblique incidence. While the latter case leads to higher proton energies due to stronger electron heating, the optimum foil target is approximately twice thinner than that found at normal incidence, a consequence of self-induced magnetostatic fields within the ion acceleration region. Secondly, we find that, by further enhancing the electron heating and mitigating the growth of the deleterious magnetostatic fields, the two-pulse scheme significantly increases the cutoff energy and number of the accelerated protons. It may also facilitate experimental investigations of RSIT-related processes by allowing thicker targets to turn relativistically transparent.

\section{Proton acceleration at normal or oblique laser incidence in relativistically transparent foils}

According to previous works (Esirkepov et al. 2006; d'Humières et al. 2013; Brantov et al. 2015), maximum ion energies attained by femtosecond laser pulses are achieved for target thicknesses close to the RSIT threshold, $l_{\text {opt }} \simeq \lambda_{0} a_{0} n_{c} / 2 n_{e}$, where $\lambda_{0}$ and $a_{0}$ are, 

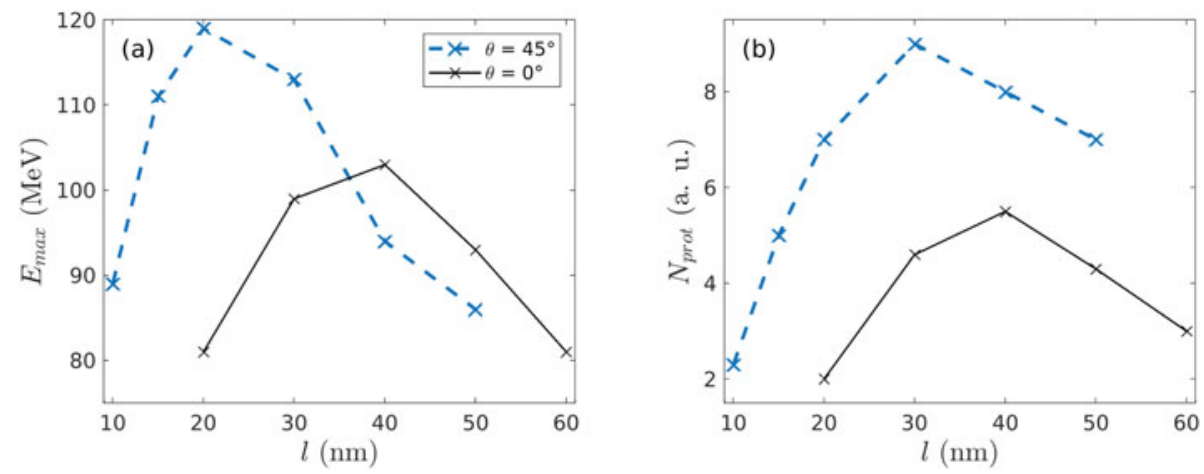

FIGURE 1. Ion acceleration by a single, $16 \mathrm{~J}$ energy, $38 \mathrm{fs}$ duration laser pulse as a function of the $\mathrm{CH}_{2}$ foil thickness: $(a)$ maximum proton energy and $(b)$ number of protons with $>30 \mathrm{MeV}$ energies. The laser pulse is either normally incident (solid black) or obliquely incident at a $45^{\circ}$ angle (dashed blue).

respectively, the laser wavelength and maximum potential vector, $n_{c}$ is the critical density and $n_{e}$ is the electron density of the target.

Our simulations have been performed in two-dimensional (2-D) geometry using the PIC code EPOCH (Arber et al. 2015). As a reference configuration, we have first considered a single laser pulse of $16 \mathrm{~J}$ energy and $0.8 \mu \mathrm{m}$ wavelength, interacting at normal incidence with a solid-density $\mathrm{CH}_{2}$ foil. The pulse is $p$-polarized (i.e. along the $y$ axis), has Gaussian transverse and temporal profiles of FWHM (full width half maximum) $w_{0}=7 \mu \mathrm{m}$ and $\tau_{0}=38 \mathrm{fs}$, respectively and a peak intensity $I_{0}=5 \times 10^{20} \mathrm{~W} \mathrm{~cm}^{-2}\left(a_{0} \simeq 15\right)$. The reference time $t=0$ corresponds to the pulse maximum reaching the target. The $\mathrm{CH}_{2}$ target is modelled as a fully ionized plasma at a $1.1 \mathrm{~g} \mathrm{~cm}^{-3}$ density, corresponding to a total electron density $n_{e}=220 n_{c}$. Its thickness, $l$, is varied in a range, $20 \leq l \leq 60 \mathrm{~nm}$, encompassing the predicted optimum, $l_{\mathrm{opt}}=30 \mathrm{~nm}$. We use 200 particles per cell and per species. The simulation domain has dimensions of $75 \times 80 \mu \mathrm{m}^{2}$ with mesh size $\Delta x \times \Delta y=4 \times 10 \mathrm{~nm}^{2}$.

Figure 1(a,b) plots (as black solid curves) the target thickness dependence of the (a) maximum proton energy and (b) the number of high-energy (above $30 \mathrm{MeV}$ ) protons, as measured at the final simulation time $\left(t_{\max }=300 \mathrm{fs}\right)$. Both quantities are found to be maximized at $l=40 \mathrm{~nm}$, close to $l_{\mathrm{opt}}$, with proton energies as high as $\sim 100 \mathrm{MeV}$ being then recorded. The number of high-energy protons is more sensitive to the target thickness than their maximum energy. Using $l=20 \mathrm{~nm}$ or $60 \mathrm{~nm}$ leads to a decrease in the proton number by a factor of 2 to 3 compared to the optimum case.

The optimum acceleration conditions are reached when RSIT occurs near the laser peak, which is confirmed by looking at the laser fields going through the target in figure 2(a-c), and by computing, for each thickness value, the transmitted $\left(C_{T}\right)$, reflected $\left(C_{T}\right)$ and absorbed $\left(C_{A}\right)$ fractions of the laser energy. While the laser pulse is significantly transmitted $\left(C_{T} \simeq 0.53\right)$ and relatively weakly absorbed $\left(C_{A} \simeq 0.12\right)$ through the $20 \mathrm{~nm}$ foil (figure $2 a$ ), it gets significantly reflected $\left(C_{R} \simeq 0.55\right)$ when the foil thickness is increased to $40 \mathrm{~nm}$ (figure $2 b$ ), while its absorption is approximately doubled $\left(C_{A} \simeq 0.23\right.$ ). If the foil thickness is further increased to $60 \mathrm{~nm}$, the laser absorption remains similar, $C_{A} \simeq 0.24$, but the transmission drops to a very low value, $C_{T} \simeq 0.04$ (figure $2 c$ ). As a result of the RSIT then taking place late in the decreasing part of the pulse, the expanding protons no longer benefit from the boost in energy caused by volumetric electron heating at the laser peak (Mishra, Fiuza \& Glenzer 2018), and hence experience a weaker overall 

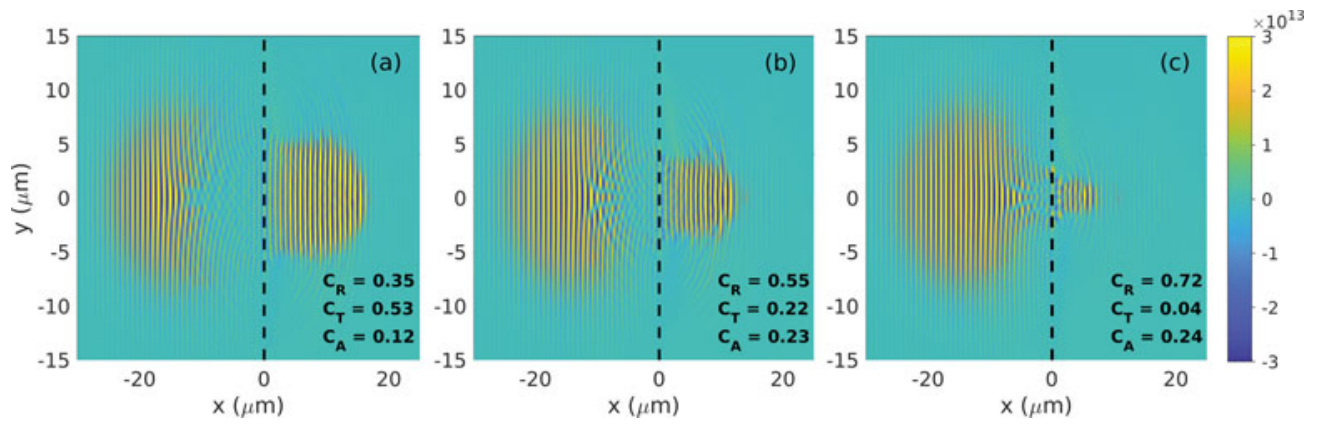

FIgURE 2. Transverse electric field $E_{y}\left(\right.$ in $\mathrm{V} \mathrm{m}^{-1}$ ) at $t=45 \mathrm{fs}$ after the on-target laser peak, for foil thicknesses $(a) l=20 \mathrm{~nm},(b) l=40 \mathrm{~nm}(b)$ and $(c) 60 \mathrm{~nm}$. In each panel the transmitted $\left(C_{T}\right)$, reflected $\left(C_{R}\right)$ and absorbed $\left(C_{A}\right)$ fractions of the laser energy are noted, and the dashed black line indicates the initial position of the foil.

acceleration. This corresponds to the transition to the TNSA regime. Note that for our parameters, and because of the foil rapidly turning transparent to the laser light, radiation pressure plays a minor role in the acceleration of the fastest ions.

The absorption processes, and therefore the onset of RSIT, are expected to be altered when operating at oblique laser incidence. To assess the dependency of ion acceleration on the incidence angle, we now consider a laser pulse impinging at an angle $\theta=45^{\circ}$ onto the foil, all other physical parameters being kept constant. The simulation domain is also enlarged to $75 \times 120 \mu \mathrm{m}^{2}$. Figure $1(a, b)$ shows (as blue dashed curves) that the optimum target thickness in terms of the maximum proton energy (respectively proton number above $30 \mathrm{MeV}$ ) then drops from $l=40 \mathrm{~nm}$ to $20 \mathrm{~nm}$ (respectively $30 \mathrm{~nm}$ ). This decrease seems reasonable since an oblique incidence angle leads to a longer path for the laser in the target, i.e. an increased effective thickness $\left(l_{\mathrm{eff}}=l / \cos \theta\right)$. More surprisingly, the decrease in the optimum target thickness goes along with a significant enhancement of the proton cutoff energy (by $\sim 15 \%$, up to $\sim 120 \mathrm{MeV}$ ) and number (by $\sim 60 \%$ ).

The improvement of the proton acceleration at oblique incidence can be explained by a more efficient mechanism for hot-electron generation. The absorption and transmission coefficients for the normal and oblique incidence are summarized in figure 3(a), showing an overall (approximately twofold) increase in the absorption coefficient at oblique incidence. For instance, the absorption coefficient at $l=40 \mathrm{~nm}$ rises from $C_{A} \simeq 0.23$ at $\theta=0^{\circ}$ to $C_{A} \simeq 0.40$ at $\theta=45^{\circ}$. By contrast, the transmission coefficient vs. thickness values are similar at $\theta=0^{\circ}$ and $45^{\circ}$ indicating that the transparency condition weakly depends on the incidence angle.

Figure $3(b)$ plots the electron energy spectra recorded $20 \mathrm{fs}$ after the on-target pulse maximum for two values of the target thickness $(l=20$ and $40 \mathrm{~nm})$. They show that the higher laser absorption found at oblique incidence translates into an increase in both the number and temperature of the hot $(>1 \mathrm{MeV})$ electrons. The thicker $(40 \mathrm{~nm})$ target seems to mainly boost the number of hot electrons in both cases.

To understand the simulation results we adapt the thin-foil expansion model proposed in Brantov et al. (2015) to the simulated scenario. This model considers the expansion of a neutral plasma slab of initial thickness $l$ and uniform ion density $n_{i 0}$, composed of a single ion species. It assumes a Boltzmann electron distribution, $n_{e}(x, t)=$ $n_{e}(0, t) \exp \left[e \phi(x, t) / T_{e}(t)\right]$, where $\phi(x, t)$ is the electrostatic potential, $T_{e}(t)$ is the (longitudinal) electron temperature and $n_{e}(0, t)$ is the (time-decreasing) electron density at the target centre $(x=0)$. The assumption of a single ion species and the choice of 

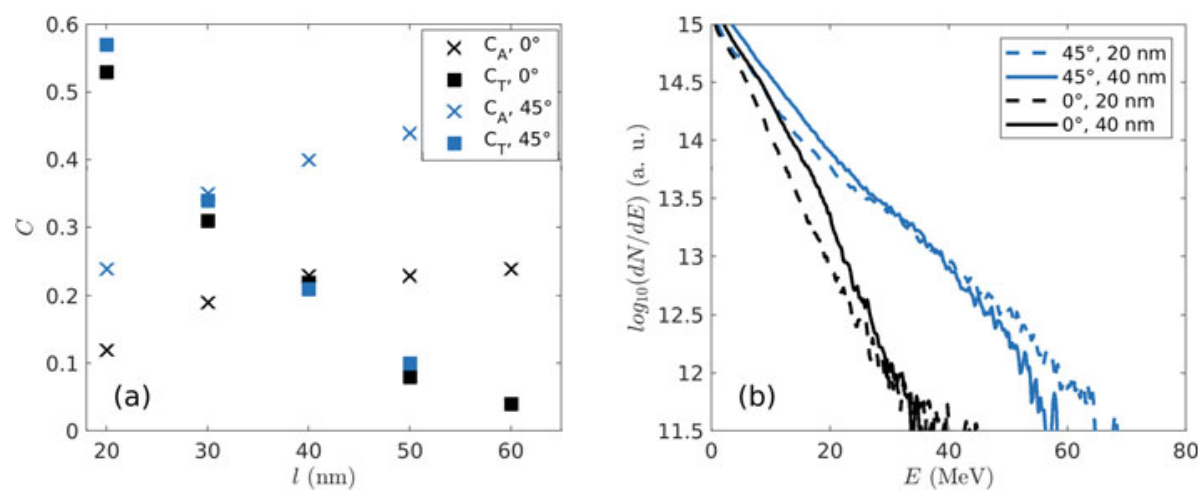

FIGURE 3. (a) Absorption ( $C_{A}$, crosses) and transmission $\left(C_{T}\right.$, squares) coefficients for normal (black) and oblique (blue) incidence as a function of the target thickness. (b) Electron energy distribution recorded at $t=20 \mathrm{fs}$ after the on-target laser peak; black and blue curves correspond to normal and oblique $\left(45^{\circ}\right)$ laser incidence, while solid and dashed curves correspond to $40 \mathrm{~nm}$ and $20 \mathrm{~nm}$ thick targets.

a Maxwell-Boltzmann distribution for the electrons may seem overly simplified, yet as it will be shown below, despite these approximations, the model is able to capture the salient features of ion acceleration from thin foils, as already observed in Brantov et al. (2015). The electrostatic field $E_{x}=-\partial_{x} \phi$ is taken to vary linearly inside the ion-filled region: $E_{x}(x, t)=E_{f} x / x_{f}(t)$ for $0 \leq x \leq x_{f}(t)$, where $x_{f}(t)$ is the position of the ion front $\left[x_{f}(0)=l / 2\right]$ and $E_{f}(t)$ is the field strength at the ion front. From this it readily follows that $\phi(x, t)=-E_{f}(t) x^{2} / 2 x_{f}(t)$. Outside the ion cloud $\left(x>x_{f}\right)$, the electric potential obeys the nonlinear Poisson equation, $\partial_{x x} \phi=e n_{e}(0, t) \exp \left(e \phi / T_{e}\right) / \epsilon_{0}$, where $e$ is the elementary charge and $\epsilon_{0}$ the vacuum permittivity. Multiplying this equation by $\partial_{x} \phi$ and integrating it over $\left[x_{f}, \infty\right]$ yields $n_{e}(0, t)=\left(\epsilon_{0} E_{f}^{2} / 2 T_{e}\right) \exp \left(e E_{f} x_{f} / 2 T_{e}\right)$. Furthermore, integrating Gauss's equation over $\left[0, x_{f}\right]$ gives

$$
\begin{aligned}
E_{f}(t) & =\frac{Z e n_{i 0} l}{2 \epsilon_{0}}-\frac{e n_{e}(0, t)}{\epsilon_{0}} \int_{0}^{x_{f}} \mathrm{~d} x \exp \left(e \phi / T_{e}\right) \\
& =\frac{Z e n_{i 0} l}{2 \epsilon_{0}}-\frac{e E_{f}^{2}}{2 T_{e}} \exp \left(e E_{f} x_{f} / 2 T\right) \int_{0}^{x_{f}} \mathrm{~d} x \exp \left(-e E_{f} x^{2} / 2 x_{f} T_{e}\right),
\end{aligned}
$$

where $Z$ is the ion charge state. Introducing $\operatorname{erf}(z)$ the error function, the above equation can be recast as

$$
E_{f}(t)=\frac{Z e n_{i 0} l}{2 \epsilon_{0}}-\frac{\sqrt{\pi} E_{f}}{2} \sqrt{\frac{e E_{f} x_{f}}{2 T_{e}}} \operatorname{erf}\left(\sqrt{\frac{e E_{f} x_{f}}{2 T_{e}}}\right)
$$

This formula assumes a 1-D expansion geometry, and hence should overestimate the field strength when the ion front has moved a distance comparable with the transverse size of the sheath field $\left(\sim w_{0} \cos \theta\right)$. A correction factor is thus applied to account for this effect

$$
E_{f, \delta}(t)=\frac{E_{f}(t)}{\left[1+\left(\frac{2 x_{f}(t)}{w_{0} \cos \theta}\right)^{2}\right]^{(\delta-1) / 2}},
$$




$\begin{array}{lrrrr}l(\mathrm{~nm}) & 20 & 30 & 40 & 50 \\ E_{\max , 0^{\circ}}(\mathrm{MeV}) & 100 & 119 & 119 & 111 \\ E_{\max , 45^{\circ}}(\mathrm{MeV}) & 139 & 156 & 156 & 151\end{array}$

TABLE 1. Maximum proton energy produced by an adaptation of the model from Brantov et al. (2015) for varying target thicknesses, at normal $\left(E_{\max , 0^{\circ}}\right)$ and oblique $\left(E_{\max , 45^{\circ}}\right)$ incidence.

where $\delta \in(2,3)$ is the spatial dimensionality of the problem $(\delta=2$ in the simulation geometry).

The electron temperature $T_{e}(t)$ is assumed to follow the rise in the laser intensity $I_{0}(t)$, here taken in the form $\sin ^{2}\left(\pi t / 2 \tau_{0}\right)$, and to stay constant in its decreasing part. The maximum electron temperature is inferred from the measured laser absorption: $T_{e, \max } / m_{e} c^{2}=0.8 C_{A}\left(a_{0}^{2} / 2\right)\left(c \tau_{0} / l \cos \theta\right)\left(n_{c} / n_{e}\right)$, where the 0.8 factor corresponds to an averaging of the intensity over the laser focal spot. After the irradiation, the electrons start cooling down adiabatically. More specifically, $T_{e}(t)$ evolves as

$$
T_{e}(t) / T_{e, \max }= \begin{cases}\sin ^{2}\left(\pi t / 2 \tau_{0}\right) & t \leq \tau_{0} \\ 1 & \tau_{0}<t \leq 2 \tau_{0}, \\ {\left[1+\left(\frac{c_{s}\left(t-2 \tau_{0}\right)}{x_{f}\left(2 \tau_{0}\right)}\right)^{\Gamma-1}\right]^{-1}} & t>2 \tau_{0} .\end{cases}
$$

We have introduced $\Gamma\left(T_{e}\right)$ the generalized adiabatic index for a relativistic 1-D gas and the typical sound speed $c_{s}=\sqrt{Z T_{e, \max } / m_{i}}$.

The knowledge of $E_{f}(t)$ allows the velocity and position of the front ions to be advanced through

$$
\begin{aligned}
\frac{\mathrm{d} v_{f}(t)}{\mathrm{d} t} & =Z e E_{f}(t) / m_{i} \\
\frac{\mathrm{d} x_{f}(t)}{\mathrm{d} t} & =v_{f}(t) .
\end{aligned}
$$

It is to be noted that the model assumes a single ion species of charge state $Z$ and mass $m_{i}=A m_{p}$ ( $m_{p}$ is the proton mass). Although the expansion dynamics of light ions may be affected by that of heavier ions in a composite target (Brantov et al. 2006), we will take $Z=1$ and $A=1$ in the following.

Equations (2.2)-(2.5) have been solved numerically for $20 \leq l \leq 50 \mathrm{~nm}$ and $\theta \in$ $\left(0^{\circ}, 45^{\circ}\right)$. The results are gathered in table 1 . The values obtained at normal incidence are quite close to those observed in the simulations - although generally slightly overestimated - and reproduce the same trend, with an optimal target thickness between 30 and 40 $\mathrm{nm}$. At oblique incidence, however, the model consistently overestimates the maximum proton energies, especially in the thicker targets $(l \geq 40 \mathrm{~nm})$. It also predicts an optimal thickness of $30-40 \mathrm{~nm}$, larger than the one $(\sim 20 \mathrm{~nm})$ observed in the simulations. This discrepancy between the simulations and the model suggests that the latter lacks a saturation mechanism that is mainly operative at oblique incidence and in thicker targets.

One such saturation-inducing effect is the generation of a strong magnetostatic field $B_{z}$ along the target surfaces (see figure $4 a, b$, associated with $l=20 \mathrm{~nm}$ and $40 \mathrm{~nm}$ ), due to the 

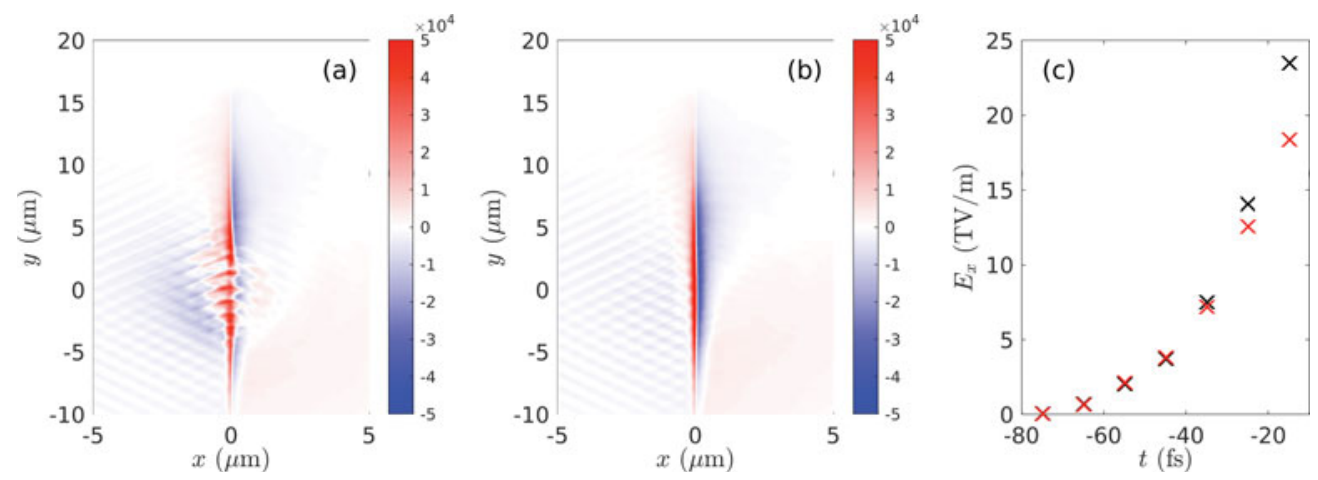

Figure 4. $(a, b)$ Magnetostatic $B_{z}$ field (in T) 10 fs before the on-target laser peak for a single obliquely incident pulse and target thicknesses $(a) l=20 \mathrm{~nm}$ and $(b) l=40 \mathrm{~nm}$. (c) Maximum of the ion-accelerating, electrostatic field $E_{x}$ on axis at the rear surface before the on-target peak, for foil thicknesses $20 \mathrm{~nm}$ (black) and $40 \mathrm{~nm}$ (red).

electron current driven in the transverse (y) direction within the plasma skin layer (Grassi et al. 2017). When volumetric electron acceleration is achieved (as is the case in the RSIT regime), this field is expected to increase with the target thickness (compare figures $4 a$ and $4 b$, where the $B_{z}$ field strength is seen to rise from $\sim 2 \times 10^{4} \mathrm{~T}$ at $l=20 \mathrm{~nm}$ to $\sim 4 \times 10^{4} \mathrm{~T}$ at $l=40 \mathrm{~nm}$ around the laser spot). This field tends to deflect the hot electrons in the transverse direction, hence limiting the growth of the on-axis electrostatic sheath field $\left(E_{x}\right)$ already before the on-target pulse maximum. This is evidenced in figure $4(c)$, which plots, for both $l=20 \mathrm{~nm}$ and $40 \mathrm{~nm}$, the temporal evolution of the maximum value of $E_{x}$ on axis at the rear surface. A similar magnetic effect has previously been shown experimentally to inhibit TNSA in micrometric targets (Nakatsutsumi et al. 2018). Therefore, although they are expected to produce stronger sheath fields, the thicker targets that turn transparent at $\theta=45^{\circ}$ do not yield faster ions, ultimately resulting in an optimum target thickness that is approximately half that found at $\theta=0^{\circ}$.

\section{Proton acceleration with colliding laser pulses in relativistically transparent foils}

We now turn to a configuration where the main laser pulse (of total energy of $16 \mathrm{~J}$ ) is split into two half-pulses of equal energy ( $8 \mathrm{~J})$ incident on the target at opposite angles $\left( \pm 45^{\circ}\right)$, similarly to the lower-intensity set-up that was lately found to boost TNSA (Ferri et al. 2019). Such a configuration could be implemented at multiple-beam laser facilities, or at single-beam facilities using split-mirror set-ups enabling spatial and temporal separation of a laser pulse (Aurand et al. 2015, 2016). In that case, to ensure overlap, the delay between the two pulses and the difference in their focus position should be small compared, respectively, with the laser duration and spot size. The other parameters remain the same as in the previous section.

The use of two half-pulses yields a larger optimal target thickness than with a single pulse, with best results obtained for $l=30-40 \mathrm{~nm}$, both regarding the maximum energy (figure $5 a$ ) and number (figure $5 b$ ) of accelerated protons. In addition, the proton maximum energy is increased from 119 to $137 \mathrm{MeV}$. Remarkably, this enhancement goes along with a 3-4-fold rise in the number of high-energy $(>30 \mathrm{MeV})$ protons. When compared with the reference case at normal incidence, the two-pulse set-up allows the maximum proton energy to be increased by $>30 \%$, and the number of energetic protons to be multiplied by a factor of 4 . Although the gain over the single obliquely incident pulse is less impressive, the predicted doubled optimal target thickness could ease the 

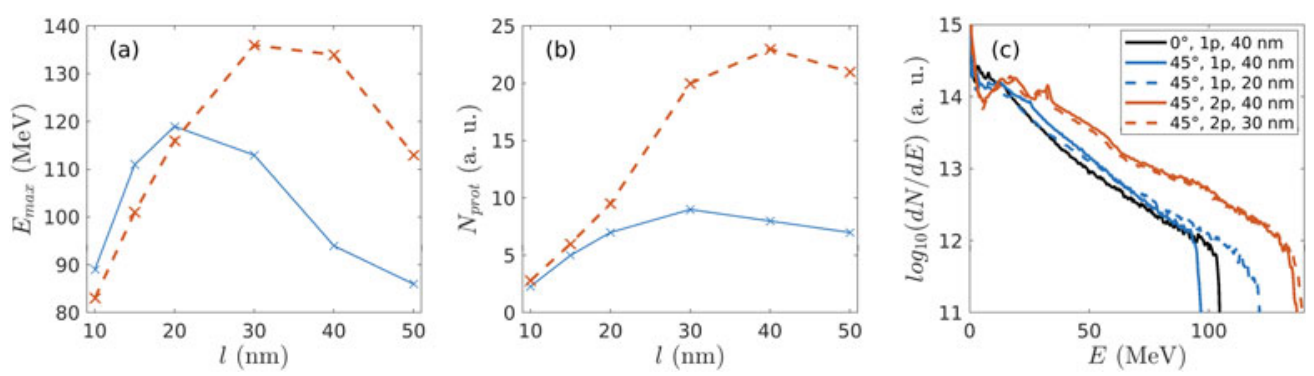

FIgURE 5. $(a, b)$ Ion acceleration by two $8 \mathrm{~J}, 38$ fs half-pulses incident at $\pm 45^{\circ}$ angles (dashed red) as a function of the $\mathrm{CH}_{2}$ foil thickness, compared to the results obtained with a single, obliquely incident $16 \mathrm{~J}$ pulse (solid blue): $(a)$ maximum proton energy and $(b)$ number of protons with $>30 \mathrm{MeV}$ energies. (c) Proton energy distributions from a $40 \mathrm{~nm}$ foil using two half-pulses (red), or a single pulse at normal (black) and oblique (blue) incidence. Solid (respectively dashed) curves correspond to $l=40 \mathrm{~nm}$ (respectively $20 \mathrm{~nm}$ ).
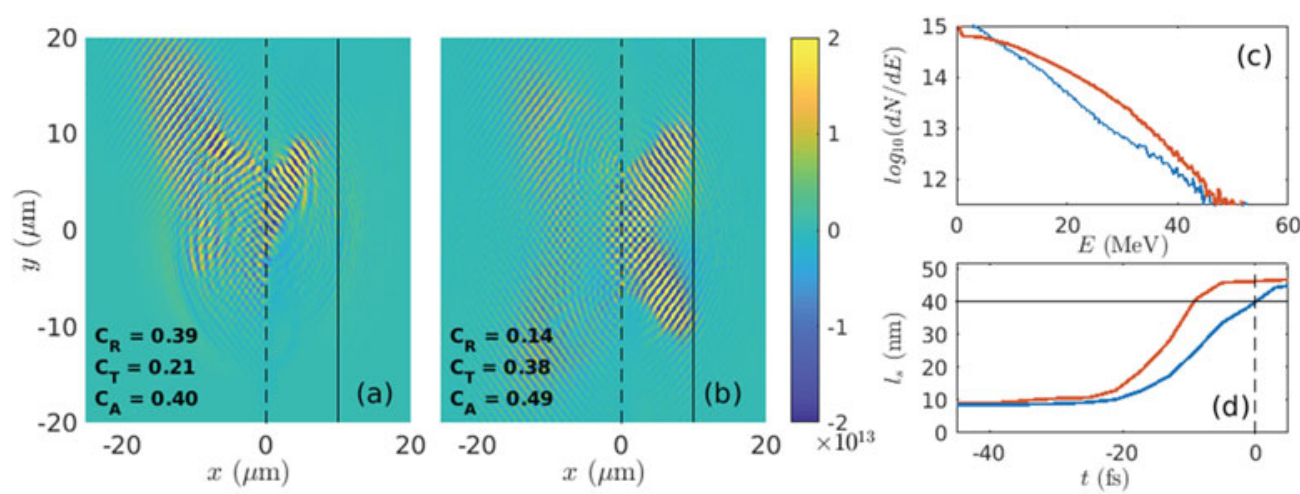

Figure 6. $(a, b)$ Transverse electric field $E_{y}$ (in $\left.\mathrm{V} \mathrm{m}^{-1}\right) 35$ fs after the on-target laser peak for a single obliquely incident pulse $(a)$ and two half-pulses $(b)$ of same $(16 \mathrm{~J})$ total energy. In both cases, the target thickness is set to $40 \mathrm{~nm}$, and the measured values of the reflection $\left(C_{R}\right)$, transmission $\left(C_{T}\right)$ and absorption $\left(C_{A}\right)$ coefficients are indicated. The dashed black line delineates the initial position of the target, while the thin grey line serves to highlight that RSIT sets in earlier in $(b)$ than in $(a)$. (c) Electron energy distribution at the peak laser intensity and (d) time evolution of the relativistic skin depth $l_{s}=\sqrt{\langle\gamma\rangle} c / \omega_{p}$ (with $\langle\gamma\rangle$ the averaged electron Lorentz factor over the laser spot) using a single (blue) or two (red) pulses. The dashed black line indicates the time of the on-target intensity peak. The solid black line indicates the initial target thickness $(40 \mathrm{~nm})$.

experimental investigation of ion acceleration from relativistically transparent solid foils, given the practical difficulties of manipulating such ultrathin targets. This applies, in particular, to modest-energy laser systems which may not allow RSIT to be achieved at handleable target thicknesses. Finally, figure 5(c) shows that the two-pulse scheme leads to substantial improvements in the proton energy spectra over the single-pulse case, both at normal and oblique incidence.

In the two-pulse scheme, the irradiated plasma area is comparable to that associated with a single pulse at oblique incidence. The larger optimum thickness and enhanced ion acceleration then mainly result from the modified electron dynamics in the two-pulse field distribution, as has been described earlier in the TNSA regime (Ferri et al. 2019). For a 


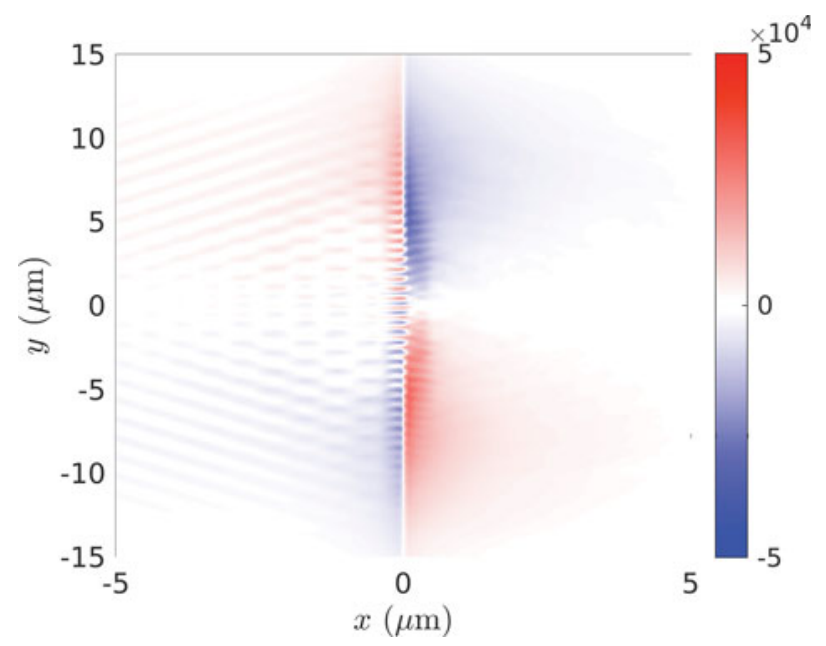

FIGURE 7. Quasistatic $B_{z}$ field (in T) $10 \mathrm{fs}$ before the on-target intensity peak for two half-pulses interacting with a $40 \mathrm{~nm}$ foil.

given target thickness, owing to the stronger electron heating, transparency is then more easily induced using two half-pulses. This is clearly shown in figure 6 , with a transmission coefficient rising from $C_{T}=0.21$ to $C_{T}=0.38$ between the single- and two-pulse cases. By examining the laser field distributions in both configurations at various successive times, we checked that RSIT occurs earlier in time using two half-pulses, a result consistent with the $\sim 25 \%$ increase in the absorption coefficient.

The stronger electron heating (see figure $6 c$ ) that accounts for the enhanced laser absorption facilitates the induction of transparency. Figure $6(d)$ shows the temporal evolution of the relativistic skin depth $l_{s}=\sqrt{\langle\gamma\rangle} c / \omega_{p}$ during the interaction, with $\omega_{p}$ the plasma frequency and $\langle\gamma\rangle$ the electron Lorentz factor spatially averaged over the target thickness and around the laser spot. As expected, the use of the two half-pulses causes a faster increase in $l_{s}$ with the laser intensity. We can consider that RSIT sets in when $l_{s}$ becomes higher than the initial target thickness $(l=40 \mathrm{~nm})$ even if this is a simplified approach as $l_{s}$ cannot entirely describe RSIT in such overdense plasmas (Cattani et al. 2000). In the two-pulse case, this criterion is fulfilled approximately 10-15 fs prior to the laser peak time, which incidentally coincides with the onset of RSIT with a single pulse. This delay between the onset times of RSIT using one or two pulses appears to be consistent with the difference between the transmitted fractions of the laser field in figure 6.

Furthermore, the symmetry (relative to the $x$ axis) of the two-pulse set-up prevents the development of a strong surface magnetostatic field. Figure 7 shows this field 10 fs before the on-target laser peak for a $40 \mathrm{~nm}$ thick target. As expected from the symmetry of the problem, the $B_{z}$ field vanishes on axis, and thus cannot counteract the sheath-field-enhancing effect of the stronger laser absorption.

\section{Conclusions}

Using 2-D PIC simulations, we have examined the efficiency of laser-driven proton acceleration in relativistically transparent, ultrathin $\mathrm{CH}_{2}$ foil targets under different short-pulse irradiation conditions. As baseline laser parameters, we have considered a $5 \times 10^{20} \mathrm{~W} \mathrm{~cm}^{-2}$ maximum intensity and a $38 \mathrm{fs}$ pulse duration. First, we have showed that 
operating at oblique incidence can be beneficial to proton acceleration, owing to enhanced energy absorption into hot electrons. Yet, the optimum target thickness $(l \simeq 20 \mathrm{~nm})$ is then reduced compared to that found at normal incidence $(l \simeq 40 \mathrm{~nm})$, as a result of strong $\left(>10^{4} \mathrm{~T}\right)$ surface magnetostatic fields self-induced within the ion acceleration region. In a second step, we have studied a configuration in which the laser pulse is split into two half-pulses with the same total energy, incoming simultaneously on target but with opposite angles of incidence. This configuration enhances the electron heating while preventing the buildup of deleterious surface $B$-fields within the laser spot. Not only does it allow one to use thicker targets than with a single obliquely incident pulse, but it also yields substantial increases in the proton numbers $(\times 4)$ and cutoff energies $(\times 1.3)$ over the baseline set-up. Our study suggests that the constraints posed by nanometric targets in experiments on RSIT could be somewhat relaxed using a two-pulse scheme.

\section{Acknowledgements}

The authors would like to acknowledge fruitful discussions with I. Thiele and L. Yi. This work was supported by the Knut and Alice Wallenberg Foundation, the Swedish Research Council, Grant No. 2016-05012, and has received funding from the European Research Council (ERC) under the European Union's Horizon 2020 research and innovation programme under grant agreement No 647121. The simulations were performed on resources at Chalmers Centre for Computational Science and Engineering (C3SE) provided by the Swedish National Infrastructure for Computing (SNIC).

Editor Luis Silva thanks the referees for their advice in evaluating this article.

\section{Declaration of interests}

The authors report no conflict of interest.

\section{REFERENCES}

Arber, T. D., Bennett, K., Brady, C. S., Lawrence-Douglas, A., Ramsay, M. G., Sircombe, N. J., Gillies, P., Evans, R. G., Schmitz, H., Bell, A. R., et al. 2015 Contemporary particle-in-cell approach to laser-plasma modelling. Plasma Phys. Control. Fusion 57 (11), 1-26.

Aurand, B., Hansson, M., Senje, L., Svensson, K., Persson, A., Neely, D., Lundh, O. \& WAHLSTRÖM, C.-G. 2015 A setup for studies of laser-driven proton acceleration at the lund laser centre. Laser Particle Beams 33 (1), 59-64.

Aurand, B., Senje, L., Svensson, K., Hansson, M., Higginson, A., Gonoskov, A., Marklund, M., Persson, A., Lundh, O., Neely, D., et al. 2016 Manipulation of the spatial distribution of laser-accelerated proton beams by varying the laser intensity distribution. Phys. Plasmas 23 (2), 023113.

Brantov, A. V., Govras, E. A., Bychenkov, V. Y. \& Rozmus, W. 2015 Ion energy scaling under optimum conditions of laser plasma acceleration from solid density targets. Phys. Rev. ST Accel. Beams 18, 021301.

Brantov, A. V., Tikhonchuk, V. T., Klimo, O., Romanov, D. V., Ter-Avetisyan, S., SChNOERRER, M., SoKollik, T. \& NicKles, P. V. 2006 Quasi-mono-energetic ion acceleration from a homogeneous composite target by an intense laser pulse. Phys. Plasmas 13 (12), 122705.

Brenner, C. M., Robinson, A. P. L., Markey, K., Scott, R. H. H., Gray, R. J., Rosinski, M., Deppert, O., BAdZiak, J., Batani, D., Davies, J. R., et al. 2014 High energy conversion efficiency in laser-proton acceleration by controlling laser-energy deposition onto thin foil targets. Appl. Phys. Lett. 104 (8), 081123.

Bulanov, S. S., Brantov, A., Bychenkov, V. Y., Chvykov, V., Kalinchenko, G., Matsuoka, T., Rousseau, P., Reed, S., Yanovsky, V., Litzenberg, D. W., et al. 
2008 Accelerating monoenergetic protons from ultrathin foils by flat-top laser pulses in the directed-coulomb-explosion regime. Phys. Rev. E 78, 026412.

Bulanov, S. V. \& KHOROShKov, V. S. 2002 Feasibility of using laser ion accelerators in proton therapy. Plasma Phys. Rep. 28, 453-456.

Cattani, F., Kim, A., Anderson, D. \& Lisak, M. 2000 Threshold of induced transparency in the relativistic interaction of an electromagnetic wave with overdense plasmas. Phys. Rev. E 62 , 1234-1237.

Ceccotti, T., Lévy, A., Popescu, H., Réau, F., D’Oliveira, P., Monot, P., Geindre, J. P., LEFEBVRE, E. \& MARTIN, P. 2007 Proton acceleration with high-intensity ultrahigh-contrast laser pulses. Phys. Rev. Lett. 99, 185002.

Clark, E. L., Krushelnick, K., Zepf, M., Beg, F. N., Tatarakis, M., Machacek, A., Santala, M. I. K., Watts, I., Norreys, P. A. \& Dangor, A. E. 2000 Energetic heavy-ion and proton generation from ultraintense laser-plasma interactions with solids. Phys. Rev. Lett. 85, $1654-1657$.

Daido, H., Nishiuchi, M. \& PirozhKov, A. S. 2012 Review of laser-driven ion sources and their applications. Rep. Prog. Phys. 75 (5), 056401.

D’Humières, E., Brantov, A., Bychenkov, V. Yu. \& Tikhonchuk, V. T. 2013 Optimization of laser-target interaction for proton acceleration. Phys. Plasmas 20 (2), 023103.

Esirkepov, T., Borghesi, M., Bulanov, S. V., Mourou, G. \& Tajima, T. 2004 Highly efficient relativistic-ion generation in the laser-piston regime. Phys. Rev. Lett. 92, 175003.

Esirkepov, T., Yamagiwa, M. \& TAJima, T. 2006 Laser ion-acceleration scaling laws seen in multiparametric particle-in-cell simulations. Phys. Rev. Lett. 96, 105001.

Ferri, J., Senje, L., Dalui, M., Svensson, K., Aurand, B., Hansson, M., Persson, A., Lundh, O., WahlströM, C.-G., Gremillet, L., et al. 2018 Proton acceleration by a pair of successive ultraintense femtosecond laser pulses. Phys. Plasmas 25, 043115.

FERRI, J., Siminos, E. \& FÜLÖP, T. 2019 Enhanced target normal sheath acceleration using colliding laser pulses. Commun. Phys. 2, 40.

Geindre, J. P., Audebert, P. \& MARJoribanks, R. S. 2006 Relativistic ac gyromagnetic effects in ultraintense laser-matter interaction. Phys. Rev. Lett. 97, 085001.

Grassi, A., Grech, M., AmiranofF, F., MaCCHI, A. \& Riconda, C. 2017 Radiation-pressure-driven ion weibel instability and collisionless shocks. Phys. Rev. E 96, 033204.

Haberberger, D., Tochitsky, S., Fiuza, F., Gong, C., Fonseca, R. A., Silva, L. O., MORI, W. B. \& Joshi, C. 2012 Collisionless shocks in laser-produced plasma generate monoenergetic high-energy proton beams. Nat. Phys. 8 (1), 95-99.

Kar, S., Kakolee, K. F., Qiao, B., Macchi, A., Cerchez, M., Doria, D., Geissler, M., McKenna, P., Neely, D., Osterholz, J., et al. 2012 Ion acceleration in multispecies targets driven by intense laser radiation pressure. Phys. Rev. Lett. 109, 185006.

MACCHI, A., Borghesi, M. \& PASSONI, M. 2013 Ion acceleration by superintense laser-plasma interaction. Rev. Mod. Phys. 85, 751.

Maksimchuk, A., Gu, S., Flippo, K., Umstadter, D. \& Bychenkov, V. Y. 2000 Forward ion acceleration in thin films driven by a high-intensity laser. Phys. Rev. Lett. 84, 4108.

Markey, K., McKenna, P., Brenner, C. M., Carroll, D. C., Günther, M. M., Harres, K., Kar, S., LAncaster, K., NÜRnberg, F., Quinn, M. N., et al. 2010 Spectral enhancement in the double pulse regime of laser proton acceleration. Phys. Rev. Lett. 105 (19), 195008.

McKenna, P., Ledingham, K. W. D., McCanny, T., Singhal, R. P., Spencer, I., Santala, M. I. K., Beg, F. N., Krushelnick, K., Tatarakis, M., Wei, M. S., et al. 2003 Demonstration of fusion-evaporation and direct-interaction nuclear reactions using high-intensity laser-plasma-accelerated ion beams. Phys. Rev. Lett. 91, 075006.

Mishra, R., FiUZA, F. \& GlenZeR, S. 2018 Enhanced ion acceleration in transition from opaque to transparent plasmas. New J. Phys. 20 (4), 043047.

Nakatsutsumi, M., Sentoku, Y., Korzhimanov, A., Chen, S., Buffechoux, S., Kon, A., Atherton, B., Audebert, P., Geissel, M., Hurd, L., et al. 2018 Self-generated surface magnetic fields inhibit laser-driven sheath acceleration of high-energy protons. Nat. Commun. 9 (1), 1-9. 
Patel, P. K., Mackinnon, A., Key, M. H., Cowan, T. E., Foord, M. E., Allen, M., Price, D. F., RUhl, H., SPRINGER, P. T. \& STEPHENS, R. 2003 Isochoric heating of solid-density matter with an ultrafast proton beam. Phys. Rev. Lett. 91, 125004.

Pelka, A., Gregori, G., Gericke, D. O., Vorberger, J., Glenzer, S. H., Günther, M. M., Harres, K., Heathcote, R., Kritcher, A. L., Kugland, N. L., et al. 2010 Ultrafast melting of carbon induced by intense proton beams. Phys. Rev. Lett. 105, 265701.

Romagnani, L., Fuchs, J., Borghesi, M., Antici, P., Audebert, P., Ceccherini, F., Cowan, T., Grismayer, T., KAR, S., MACCHI, A., et al. 2005 Dynamics of electric fields driving the laser acceleration of multi-mev protons. Phys. Rev. Lett. 95, 195001.

Roth, M., Cowan, T. E., Key, M. H., Hatchett, S. P., Brown, C., Fountain, W., Johnson, J., Pennington, D. M., Snavely, R. A., Wilks, S. C., et al. 2001 Fast ignition by intense laser-accelerated proton beams. Phys. Rev. Lett. 86, 436-439.

Scott, G., Green, J., Bagnoud, V., Brabetz, C., Brenner, C., Carroll, D., Maclellan, D., Robinson, A., Roth, M., Spindloe, C., et al. 2012 Multi-pulse enhanced laser ion acceleration using plasma half cavity targets. Appl. Phys. Lett. 101 (2), 024101.

Silva, L. O., Marti, M., Davies, J. R., Fonseca, R. A., Ren, C., Tsung, F. S. \& Mori, W. B. 2004 Proton shock acceleration in laser-plasma interactions. Phys. Rev. Lett. 92, 015002.

Snavely, R. A., Key, M. H., Hatchett, S. P., Cowan, T. E., Roth, M., Phillips, T. W., Stoyer, M. A., Henry, E. A., SAngster, T. C., Singh, M. S., et al. 2000 Intense high-energy proton beams from petawatt-laser irradiation of solids. Phys. Rev. Lett. 85, 2945-2948.

Tikhonchuk, V. 2010 Physics of laser-assisted ion acceleration. Nucl. Instrum. Meth. Phys. Res. Sect. A $620(1), 1-13$.

Wilks, S. C., Langdon, A. B., Cowan, T. E., Roth, M., Singh, M., Hatchett, S., Key, M. H., Pennington, D., MacKinnon, A. \& Snavely, R. A. 2001 Energetic proton generation in ultra-intense laser-solid interactions. Phys. Plasmas 8 (2), 542.

Yin, L., Albright, B. J., Hegelich, B. M. \& Fernández, J. C. 2006 GeV laser ion acceleration from ultrathin targets: the laser break-out afterburner. Laser Particle Beams 24 (2), 291-298.

Zeil, K., Kraft, S. D., Bock, S., Bussmann, M., Cowan, T. E., Kluge, T., Metzkes, J., Richter, T., SAUERbrey, R. \& SChramm, U. 2010 The scaling of proton energies in ultrashort pulse laser plasma acceleration. New J. Phys. 12 (4), 045015. 